\title{
GENDER DIFFERENCES IN THE HUMAN CERVICOTHORACIC GANGLIA
}

\author{
Anita Dabuzinskiene ${ }^{1}$, Aleksandras Ratkevicius ${ }^{1}$, \\ Darius Batulevicius ${ }^{1}$, Gertruda Skripkiene ${ }^{1}$, Valdas Skripka ${ }^{1}$, \\ Gineta Liutkiene ${ }^{1}$, Ugnius Algirdas Bagdonas ${ }^{2}$, Sigita Razbadauskiené ${ }^{3}$ \\ ${ }^{1}$ Institute of Anatomy, Medical Academy, \\ Lithuanian University of Health Sciences, Kaunas \\ ${ }^{2}$ Medical Faculty, Medical Academy, Lithuanian University of Health Sciences \\ ${ }^{3}$ Kaunas Republic Hospital
}

\begin{abstract}
Researchers still further have a morphological interest in sympathetic ganglia. Functionally, paravertebral ganglia are involved in many physiological and pathological aspects of neuropathic, vascular, visceral pain syndromes, the Raynaud's syndrome and hyperhydrosis. Several interventions on ganglia as surgical and chemical sympathectomy, the ganglion block, and the chemical or thermal sympatholysis are applied for treatment of the pathological conditions. Accurate knowledge about the structure and the location of ganglia is required for a successful after effect of these procedures. In scientific literature there are many facts about the structural variations of the sympathetic ganglia related to development, age, pathology, lateral asymmetry and gender. The last one is the indeterminate factor, and the data about it are few and controversial. Still we were missing the information about the gender role on morphometry of human sympathetic ganglia and particularly of the cervicothoracic ganglion. The goal of our present study was to evaluate gender differences in the human ganglia. In our study we found that male cervicothoracic ganglia were longer than female ganglia, $21.33 \pm 4.74 \mathrm{~mm}$ vs. $14.87 \pm 1.84 \mathrm{~mm}$, wider $9.51 \pm 1.48 \mathrm{~mm}$ vs. $8.76 \pm 1.14$ $\mathrm{mm}$, and thicker than female ganglia $5.19 \pm 0.77$ vs. $4.29 \pm 0.36 \mathrm{~mm}$. The dissected ganglia exhibited the three main distinguishable shapes: spindle, dumbbell and inverted "L". We defined gender differences: the female ganglia were mainly of spindle shape $(78 \%)$, whereas the male ones equally expressed all the three types of the shape $(35 \%, 30 \%$, and
\end{abstract}


$35 \%$ ). In summary, we determined the gender differences in human cervicothoracic ganglia. We note that these differences are important for interventions on ganglia.

Key words: Cervicothoracic ganglion, human, gender, anatomy.

\section{INTRODUCTION}

Researchers still further have a morphological interest in sympathetic ganglia. Functionally, paravertebral ganglia are involved in many physiological and pathological aspects of neuropathic, vascular, visceral pain syndromes, the Raynaud's syndrome and hyperhydrosis. Several interventions on ganglia- surgical and chemical sympathectomy, the ganglion block, and chemical or thermal sympatholysis are applied as a possible treatment for pathological conditions [2]. The stellate ganglion block decreases the cerebral vascular tone for relieving cerebral vasospasm [4]. Accurate knowledge about the structure and the location of ganglia is required for a successful after effect of these procedures. In scientific literature there are many facts about structural variations of the sympathetic ganglia related to development, age, pathology, lateral asymmetry and gender. The last one is the indeterminate factor and the data about it are few and controversial. There were gender differences on the frequency of neuroaxonal dystrophy in diabetic human autonomic ganglia. Frequency was increased threefold in men in comparison to women [9]. Electrophysiological investigations had revealed the gender related differences in autonomic cardiac control and in clinical expressions of ganglia functions, such as human females are more prevalent to a variety of chronic pain than males [8]. The cadaveric studies determined pathology- dependent histological changes on the left stellate ganglion [3]. Still we were missing the information about the gender role on the morphometry of human sympathetic ganglia and particularly of the cervicothoracic ganglion.

The goal of our present study goal was to evaluate gender differences in the human cervicothoracic ganglia. 


\section{MATERIALS AND METHODS}

We examined 41 cervicothoracic ganglia of 26 autopsied human subjects within 18-24 h delay. This study was approved by the Kaunas Regional Bioethics Committee. There were 15 males and 10 females, aged between 60 and 80 years (the mean age at death was 75 years). The cadavers displaying the pathology of the nervous system, traumatic lesions and surgical procedures in the neck were excluded. In supine the cadaver's position in the middle line of the neck skin incision was done. The sternocleidomastoid muscle was dissected and pushed laterally for the exposition of the carotid vagina. Soft tissue and muscles of the neck were removed, and subclavian and vertebral arteries were dissected. As skeletal landmarks for the dissection, the transverse process of the seventh cervical vertebra and the neck of the first rib were used [5]. Removed ganglia were grouped by gender and the side (right, left) (Table 1).

Table 1. Groups of human cervicothoracic ganglia.

\begin{tabular}{|l|c|c|c|}
\hline Ganglia/Gender & Female & Male & Total \\
\hline Right cervicothoracic ganglia & 10 & 13 & 23 \\
\hline Left cervicothoracic ganglia & 8 & 10 & 18 \\
\hline Total & 18 & 23 & 41 \\
\hline
\end{tabular}

The ganglia measurements: length, width, and thickness were made by using outside callipers (sensitivity $0.01 \mathrm{~mm}$ ) and recorded in a specially created pro forma. The types of shapes were defined: spindle, dumbbell and inverted "L" by the appearance of the spindle, the constriction or the waist and the hook like form [7]. During dissection on ganglia the photographs were taken by the camera Fujifilm Finepix JV150.

Prepared cervicothoracic ganglia were fixed in paraformaldehyde 4\% buffer solution and embedded in paraffin. For light microscopy $7 \mu \mathrm{m}$ thick serial sections were cut and stained with cresylviolet for the histological confirmation of ganglia. Statistic evaluation was performed by using SSPS 13.0 software. Data differences were considered significant if the level of significance was at $p<0.05$. 


\section{RESULTS}

Macroscopically, human cervicothoracic ganglia were greyish, enclosed in the connective tissue capsule, surrounded by fascia, adipose tissues and lymphatic nodes. Topographically, the ganglion was located close to the lateral border of the longus colli muscle. Above and laterally of it, we found the inferior trunk of brachial plexus and the anterior scalene muscle. The ganglion was located behind the vertebral artery. In all 26 dissections the cervicothoracic ganglion was formed by the fusion of two ganglia: the inferior cervical and the first thoracic. We did not dissect one right-sided and 4 left-sided ganglia because of the pathologies in the neck and the upper part of the thorax. The obtained dimensional results were tabulated in Tables $2,3,4,5$. We found that the mean length of the right and the left cervicothoracic ganglia were $19.05 \pm 3.62 \mathrm{~mm}$ and $17.41 \pm 2.68 \mathrm{~mm}$, respectively. The mean total length for all the ganglia was $18.30 \pm 2.17 \mathrm{~mm}$ (Table 2).

Table 2. Length, width and thickness of cervicothoracic ganglia.

\begin{tabular}{|l|c|c|c|c|}
\hline Variable & Ganglia (n) & Length (mm) & Width (mm) & Thickness (mm) \\
\hline Left & 18 & $17.41 \pm 2.68$ & $8.65 \pm 1.47$ & $4.56 \pm 0.51$ \\
\hline Right & 23 & $19.05 \pm 3.62$ & $9.15 \pm 0.93$ & $4.93 \pm 0.52$ \\
\hline $\begin{array}{l}\text { Left, } \\
\text { right }\end{array}$ & 17 & $17.55 \pm 2.18$ & $9.21 \pm 0.80$ & $4.78 \pm 0.42$ \\
\hline Total & 41 & $18.30 \pm 2.17$ & $8.92 \pm 0.79$ & $4.76 \pm 0.35$ \\
\hline Male & 10 & $19.29 \pm 6.02$ & $9.29 \pm 2.31$ & $5.14 \pm 1.14$ \\
\hline Left & 13 & $23.36 \pm 5.65$ & $9.73 \pm 1.68$ & $5.23 \pm 0.88$ \\
\hline Right & 23 & $21.33 \pm 4.74$ & $9.51 \pm 1.48$ & $5.19 \pm 0.77$ \\
\hline Total & 8 & $15.10 \pm 2.38$ & $9.20 \pm 1.68$ & $4.15 \pm 0.43$ \\
\hline Female & 10 & $14.63 \pm 2.32$ & $8.31 \pm 0.78$ & $4.38 \pm 0.50$ \\
\hline Left & 18 & $14.87 \pm 1.84$ & $8.76 \pm 1.14$ & $4.29 \pm 0.36$ \\
\hline Right & Total & \multicolumn{5}{|l|}{} \\
\hline
\end{tabular}

Table 3. Incidence of different shapes of cervicothoracic ganglia.

\begin{tabular}{|l|c|c|c|c|c|c|}
\hline Shape & Left & $\mathrm{n}$ & Right & $\mathrm{n}$ & Total & $\mathrm{n}$ \\
\hline Spindle & $\mathbf{5 5 \%}$ & 11 & $\mathbf{5 7 \%}$ & 12 & $\mathbf{5 6 \%}$ & 23 \\
\hline Dumbbell & $\mathbf{2 5 \%}$ & 5 & $\mathbf{2 4 \%}$ & 5 & $\mathbf{2 4 \%}$ & 10 \\
\hline Inverted "L" & $\mathbf{2 0 \%}$ & 4 & $\mathbf{1 9 \%}$ & 4 & $\mathbf{2 0 \%}$ & 8 \\
\hline
\end{tabular}


Table 4. Incidence of different shapes of male cervicothoracic ganglia.

\begin{tabular}{|l|c|c|c|c|c|c|}
\hline Shape & Left & $\mathrm{n}$ & Right & $\mathrm{n}$ & Total & $\mathrm{n}$ \\
\hline Spindle & $\mathbf{3 0 \%}$ & 3 & $\mathbf{3 8 \%}$ & 5 & $\mathbf{3 5 \%}$ & 8 \\
\hline Dumbbell & $\mathbf{3 0 \%}$ & 3 & $\mathbf{3 1 \%}$ & 4 & $\mathbf{3 0 \%}$ & 7 \\
\hline Inverted "L" & $\mathbf{4 0 \%}$ & 4 & $\mathbf{3 1 \%}$ & 4 & $\mathbf{3 5 \%}$ & 8 \\
\hline
\end{tabular}

Table 5. Incidence of different shapes of female cervicothoracic ganglia.

\begin{tabular}{|l|c|c|c|c|c|c|}
\hline Shape & Left & $\mathrm{n}$ & Right & $\mathrm{n}$ & Total & $\mathrm{n}$ \\
\hline Spindle & $\mathbf{8 8 \%}$ & 7 & $\mathbf{7 0 \%}$ & 7 & $\mathbf{7 8 \%}$ & 14 \\
\hline Dumbbell & $\mathbf{1 2 \%}$ & 1 & $\mathbf{2 0 \%}$ & 2 & $\mathbf{1 7 \%}$ & 3 \\
\hline Inverted "L" & $\mathbf{0 \%}$ & 0 & $\mathbf{1 0 \%}$ & 1 & $\mathbf{5 \%}$ & 1 \\
\hline
\end{tabular}

Male cervicothoracic ganglia were longer than female ganglia, $21.33 \pm 4.74 \mathrm{~mm}$ vs. $14.87 \pm 1.84 \mathrm{~mm}$, wider $9.51 \pm 1.48$ vs. $8.76 \pm$ $1.14 \mathrm{~mm}$, and thicker than female ganglia $5.19 \pm 0.77$ vs. $4.29 \pm 0.36 \mathrm{~mm}$. The dissected ganglia exhibited the three main distinguishable shapes: spindle, dumbbell and inverted "L" (Table 3), (Figure 1). The largest incidence was for the spindle shape, the smallest incidence was for inverted "L" shape ganglia. These results were obtained on both rightand left-sided ganglia. We defined the gender differences of ganglia. The female ganglia were mainly of spindle shape (78\%; Table 4), whereas the male ones equally expressed all the tree types of shape $(35 \%, 30 \%$, and $35 \%$; Table 5).

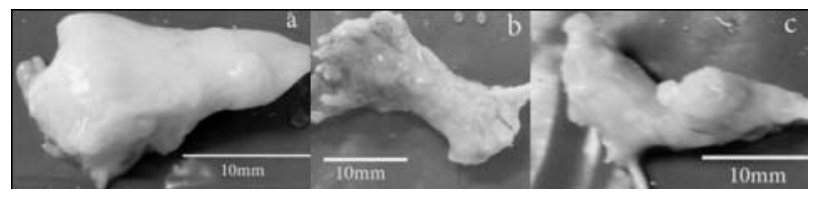

Figure 1. Shapes of human cervicothoracic ganglia: $a-$ spindle; $b-$ dumbbell; c - inverted "L" shape.

\section{DISCUSSION}

Saylam C.Y. et al. [10] examined 40 inferior cervical/cervicothoracic ganglia: $70 \%$ were located behind the vertebral artery, $25 \%$ posterolaterally and only $5 \%$ posteromedially to the vertebral artery. Sceletotopically, $40 \%$ of inferior cervical/cervicothoracic ganglia were 
located at the level of the seventh cervical vertebra. Fifty-five per cent of inferior cervical/cervicothoracic ganglia were located more caudally: at the level of the intervertebral disc between the seventh cervical/first thoracic vertebra $(25 \%)$ and at the level of the first thoracic vertebra $(35 \%)$. Ateş Y. [1], using cadaver material and MRI scans, revealed that the longus colli muscle is an important landmark structure for the cervicothoracic ganglion block, and males have a thicker muscle at each vertebral level. We observed the similar position of ganglia. The means of the length and the width of ganglia were $11.3 \pm 4.6 \mathrm{~mm}(5.1-23 \mathrm{~mm})$ and $8.2 \pm 3.0 \mathrm{~mm}(3.5-15.6 \mathrm{~mm})$ of 20 male cadavers, respectively [10]. Our measurements were correspondingly $18.30 \pm 2.17 \mathrm{~mm}(7-44 \mathrm{~mm})$; $8.92 \pm 0.79 \mathrm{~mm}(1-15)$. We suggest that the cause for the disagreement in results is that we made the measurements of ganglia from individuals of both genders of precisely known age, whereas Saylam C.Y. could not determine the exact age and did not publish the data about gender differences. Zhang B. et al. [11] in their protocol defined the length $19.3 \pm 2.3 \mathrm{~mm}(14.7-25.2)$, and the width $6.5 \pm 1.7 \mathrm{~mm}(4.1-8.5 \mathrm{~mm})$ of human ganglia. For this study the material of 18 males and 7 females was used, but the authors did not give scientific data about it and the exact age. Conditionally we can consider that our obtained findings are similar. One more factor that is important for the rate of results is that Saylam C.Y. and Zhang B. et al. used cadavers already embalmed and fixed with the formaldehyde solution. Kalsey G. et al. [6] reported the average dimensions of human cervicothoracic ganglia: $2.0 \times 1.0 \times 0.3 \mathrm{~cm}$. This group of researchers identified that ganglions in all the cases were the fusion of inferior cervical and the first thoracic ganglions. They made a set of ganglia shapes: oval, stellate, hourglass, elongated, comma, C or the club shaped. In the study of Pather N. et al. [7] we found a detailed morphological description of human ganglia. In comparison with our findings, Pather N. et al. [7] detected more inverted shape ganglia ( $45.3 \%$ vs. $20 \%)$ and less spindle shape ganglia $(28 \%$ vs. $56 \%$ ). The incidence of dumbbell shape is similar between Pather N. et al. [7] and our study ( $26.7 \%$ vs. $24 \%)$. Pather's study material was 48 human subjects: 31 fetuses (the mean age 34 weeks) and 17 adult subjects (the mean age 48 years). There is a wide range of age in the study of Pather N. et al. [7], and many factors may influence the structure of human ganglia during the developmental changes. 
In summary, we determined the significant morphometric differences in the cervicothoracic ganglion between the male and the female. These gender differences may be important for surgical and physiological interventions on the human sympathetic ganglia.

\section{REFERENCES}

1. Ateş Y., Ozgencil E., Açar HI., Yağmurlu B., Tekdemir I. (2009) Evaluation of the longus colli muscle in relation to stellate ganglion block. Reg Anesth Pain Med, 34, 3, 219-223.

2. Day M. (2008) Sympathetic blocks: the evidence. Pain practice, 8, 2, 98-109.

3. Docimo S., Piccolo C., Van Arsdale, Elkowith D.E. (2008) Pathologydependent histological changes of the left stellate ganglion: a cadaveric study. Clinical Medicine: Pathology, 1, 105-108.

4. Gupta M.M., Bithal P.K., Dash H.H., Chaturvedi, Mahajan R.P. (2005) Effects of stellate ganglion block on cerebral haemodinamics as assessed by transcranial doppler ultrasonography. Br J Anaesth, 95, 5, 669-673.

5. Janik Je., Hoeft MA., Ajar AH., Alsoform GF., Borrello MT., Rathmell JP. (2008) Variable osteology of the sixth cervical vertebra in relation to stellate ganglion block. Reg Anesth Pain Med, 33, 2, 102-108.

6. Kalsey G., Mukherjee R.N., Patniak V.V.G. (2000) A comparative study of cervical sympathetic chain. J Anat Soc India, 49, 1, 26-30.

7. Pather N., Partab P., Singh B., Satyapal K.S. (2006) Cervico-thoracic Ganglion: its clinical implications. Clinical Anatomy, 19, 323-326.

8. Riley J., III, Robinson M., Wise EA., Myers CD., Fillingim RB. (1998) Sex differences in the perception of noxious experimental stimuli: a meta-analysis. Pain, 74, 2-3, 181-187.

9. Schmidt R.E., Plurad S.B., Parvin C.A., Roth K.A. (1993) Effect of diabetes and aging on human sympathetic autonomic ganglia. Am $\mathbf{J}$ Pathol, 143, 1, 143-153.

10. Saylam C.Y., Ozgiray E., Orhan M., Cagli S., Zizeli M. (2009) Neuroanatomy of cervical sympathetic trunk: a cadaveric study. Clinical Anatomy, 22, 324-330.

11. Zhang B., Li Z., Yang X., Li G., Wang Y., Cheng J., Tang X., Wang F. (2009) Anatomical variations of the upper thoracic sympathetic chain. Clinical Anatomy, 22, 595-600. 
Address for correspondence:

Anita Dabuzinskiene,

Lithuanian University of Health Sciences

Institute of Anatomy, Medical Academy

Mickeviciaus 9, Kaunas, LT-44307, Lithuania

E-mail: anitadab@1smuni.lt 